\title{
Kardinal Königs Kommunikationsstrategie
}

\author{
von John W. Mole
}

Eine Karikatur in der Torontoer Zeitung "Globe and Mail“ vom 24. August 1967 zeigt einen Anschlag: „Heute Theologenkongreß - McLuhan spricht - $20 \mathrm{Uhr}-$ Convocation Hall.“ Z $Z$ wei Geistliche gehen vorüber, und der eine sagt zum anderen: „Gott weiß, worüber er sprechen wird, ich glaub's.“

Doch nicht der berühmte Professor der Universität von Toronto sprach vor dem "Kongreß für theologische Erneuerung" in Toronto am 24. August über die Kommunikationsmedien, sondern Kardinal König aus Wien. Der angekündigte Vortrag war denn auch mit „Theologie der Kommunikation“ überschrieben, und McLuhan hätte gewiß nicht über einen offensichtlich außerhalb seiner Kompetenz liegenden Gegenstand gesprochen. Sprach der Kardinal nun klarer über dieses Thema, als McLuhan es hätte tun können? Eher kann man sagen: Er mied es völlig und sprach stattdessen über eine von ihm so bezeichnete "Kommunikationsstrategie“. Er empfahl dringend, daß Geistliche sich für die in den letzten dreißig Jahren in den USA entwickelte Kommunikationsforschung interessierten, wodurch nach seiner Ansicht die Strategie der Kirche in der Kommunikation mit den modernen Menschen gefördert werden könnte.

Der Wiener Kardinal machte eine flüchtige Anspielung auf die Vortrefflichkeit der Studien Marshall McLuhans, wobei er vielleicht nicht bemerkte, daß der in Rede stehende katholische Wissenschaftler die amerikanische Schule der Kommunikationsforschung weitgehend ablehnt, weil sie sich zu sehr mit dem Inhalt der Kommunikation beschäftige. Das Medium selbst, nicht der Inhalt, sollte nach McLuhan die „Botschaft" sein, das heißt: im Brennpunkt des Interesses stehen, zumindest was die Kommunikationsforschung angeht.

Wilbur Schramm, der in den fünfziger Jahren vom Nationalen Kirchenrat der Vereinigten Staaten (U.S. National Council of Churches) beauftragt wurde, ein Buch über Verantwortung in der Massenkommunikation („Responsibility in Mass Communication"1) zu schreiben, war Herausgeber der 1963 veröffentlichten Berichte eines Symposiums über die Wissenschaft von der menschlichen Kommunikation. ${ }^{2}$ Dort nennt er als Begründer dieser amerikanischen Forschungsrichtung folgende Wissenschaftler: Paul Lazarsfeld, in Wien ausgebildeter Soziologe; Harold Lasswell, Politikwissenschaftler; Kurt Lewin, Gestaltpsychologe aus Wien; und Carl Hovland, Experimentalspychologe, der im Zweiten Weltkrieg in der Propagandaforschung für die amerikanische Regierung tätig war.

John W. Mole OMI ist Direktor der School of Communications an der St. Paul University in Ottawa/Kanada. Er ist Herausgeber der Zeitschrift "Christian Communications", Ottawa. Seine Stellungnahme zu Kardinal Königs Vortrag in Toronto "Theology of Communications" (vgl. Abdruck unter dem Titel „Kirche und Kommunikation" in CS Nr. 1/1. Jg. 1968, S. 6-20) führt nur einige Teilgegenstände aus Königs für ein neues kirchliches Publizistik-Verständnis fundamentaler Darlegung in die Arena der Diskussion. Er macht deutlich, daß es unter den Theologen noch erheblich stärkerer Anteilnahme an diesem Thema bedarf, wenn der beliebte Begriff Dialog nicht auf einem wichtigen Gebiet leere Hülse bleiben soll. Das Forum dafür stellt CS gern zur Verfügung. 
Lazarsfeld gründete ein Forschungsinstitut an der Columbia Universität, und einer seiner Schüler, Joseph T. Klapper, gewann hohes Ansehen als tüchtiger Historiker und Interpret der Tradition der amerikanischen Kommunikationsforschung. Sie hat bis heute sehr wenig erreicht, so lautet Klappers Urteil in seinem Buch „The Effects of Mass Communication"3 (Die Wirkungen der Massenkommunikation), welches weitgehend das Eingeständnis impliziert, daß sämtliche Bemühungen einer neuen Orientierung bedürfen.

Die Frage, die sich in diesem Zusammenhang stellt, lautet: Was war falsch an der ursprünglichen Orientierung dieser besonderen Schule der Kommunikationsforschung? Es wäre für die Kirche offensichtlich unklug, irgendeine „Kommunikationsstrategie“ auf eine Forschungsart zu stützen, die sich nicht als vernünftig orientiert erwiesen hat.

Aus Raumgründen können wir nur einen von mehreren Gründen, dazu in ziemlich vereinfachter Form, angeben. Doch erfüllt das unseren gegenwärtigen $Z$ weck, den Theologen zu empfehlen, sich erst kritisch umzusehen, bevor sie Kardinal Königs Vorschlag folgen. Der Grund ist folgender: Die amerikanische Form der Kommunikationsforschung erhielt ihre ursprüngliche Orientierung und ihren ersten Anstoß durch das Kriegsministerium der Vereinigten Staaten während des Zweiten Weltkrieges. Wissenschaftler der Universitäten wurden mobilisiert, den Inhalt von Feindpropaganda $\mathrm{zu}$ analysieren und eine Strategie der Gegen-Propaganda im Sinne der "Psychologischen Kriegsführung“ zu entwerfen. Aus diesen Bemühungen erwuchs der wichtigste Bereich der amerikanischen empirischen Kommunikationsforschung, die "Aussage-Analyse“.

Nach dem Kriege wurden die amerikanischen Kommunikationsforschungsprojekte von den Werbeagenturen und den Verkaufsabteilungen der Industrie finanziell unterstützt. Benutzt wurde die Psychologie des Behaviorismus, deren Begründer, John B. Watson, auch Präsident einer Werbeagentur war. Ernest Dichter, ein weiterer Wiener Psychologe, und sein Institut für Motivforschung hatten ebenfalls bedeutenden Anteil an der Entwicklung einer Werbung, welche die Prosperität einer verbraucherorientierten Wirtschaft sicherstellen konnte. Die Strategie Dichters zielte auf enorme Erhöhung des Konsums von "besseren Dingen zu besserem Leben“ $a b$, indem sie zu Assoziationen mit der Befriedigung sexueller Wünsche, der StatusSuche etc. hinlenkte. Die durchgängige Absicht dieser Strategie besteht darin, den amerikanischen Käufer von jüdisch-christlichen Attitüden abzubringen, welche ihn, so klagt Dichter, zu sehr zu Sparsamkeit und Mäßigung im Gebrauch der Güter dieser Welt bestimmen.

Das Problem der amerikanischen Kommunikationsforschung lautet: Wie soll sie sich umorientieren, um ein Unternehmen zum echten Vorteil der gesamten Gesellschaft zu werden, statt weiterhin immer einfallsreichere Methoden zur Manipulation der Menschen im Interesse der Konsumgüterindustrie zu erforschen?

Es besteht Hoffnung, daß der Ruf nach einer solchen Umorientierung aus der Pädagogik kommen wird. Beispielsweise macht sich der sehr ernste Wunsch nach Forschungen über die Wirkung von Massenmedien auf Kinder bemerkbar. Ist es ihrer Erziehung förderlich oder hinderlich, wenn sie den Medien massiv ausgesetzt sind?

Schramm selbst hat in der Tat eine Forschungsbewegung in dieser Richtung inauguriert und zusammen mit anderen Autoren einen Überblick verfaßt, der unter dem Titel "Television in the Lives of Our Children " (Fernsehen im Leben unserer Kinder) veröffentlicht wurde. Viele Erzieher hätten es gewiß begrüßt, wenn 
Kardinal König die Universitäten gedrängt hätte, sich ernsthaft vorzunehmen, eine bessere Form der Kommunikationsforschung aufzubauen als diejenige, welche wirksamere Propagandatechniken $\mathrm{zu}$ entwickeln sucht.

Jedenfalls zog Kardinal König es vor, den größten Teil seines Vortrags Problemen der Sprache zu widmen statt der amerikanischen Kommunikationsforschung. Er begann mit den Ansichten des heiligen Augustinus über die „Kunst der Verkündigung“, unterstrich einigermaßen ausführlich die Bedeutung der „Kunstfertigkeit in der Kommunikation", und gelangte schließlich zu dem schwierigen Problem, welches die Schaffung einer inter-disziplinären Sprache darstellt, mit Hilfe derer sich Wissenschaftler verschiedener Gebiete untereinander verständigen könnten. Sodann unternahm er einen recht langen Ausflug in das Gebiet des logischen Positivismus und erging sich schließlich in Spekulationen über die etwaige Möglichkeit einer Sprache, in der Theologen und Philosophen sich dem Gespräch anschließen könnten, welches Naturwissenschaftler eines Tages unter sich beginnen könnten.

Die Probleme der Sprache sind Forschungsgegenstand, seit die alten Griechen für Grammatik, Syntax, Rhetorik und Logik Regeln aufstellten. Nachdem einige neue Disziplinen hinzugekommen sind, z. B. die Semantik, scheint dieses Studiengebiet die allgemeine Bezeichnung einer "Kommunikationskunst" $z u$ erwerben. Seine Adepten beschäftigen sich indes mit Problemen des Ausdrucks" und nicht der "Kommunikation" im eigentlichen Sinne. Ausdruck ist eine individuelle Leistung, während Kommunikation im wesentlichen ein kollektiver oder sozialer Vorgang ist und daher der Untersuchung einen völlig verschiedenen Gegenstand darbietet. Sie kann, wie die Ehe, nur stattfinden, wenn zwei oder mehr Personen sich in einer gemeinsamen Situation zusammenfinden. „Kommunizieren" heißt: etwas gemeinsam haben oder unternehmen. Kardinal König brachte bestimmte Gedanken in der Convocation Hall von Toronto am Abend des 24. August 1967 zum Ausdruck. Der Text seiner Rede wurde der Presse zugeleitet und kann daher in seinem Inhalt studiert werden. Doch ob diese Gedanken wirklich kommuniziert wurden, das heißt, von allen oder einem Teil der 2000 Personen, die zur gleichen Zeit wie der Kardinal in der Halle waren, mitteilungsweise angenommen wurden, ist eine ganz andere Frage und erfordert eine ganz andere Art der Untersuchung.

Außerdem beschäftigt sich Kommunikationsforschung nicht so sehr mit dem sozialen Verkehr im allgemeinen als mit den Phänomenen und Problemen, die sich aus der Anwendung der elektronischen Technologie auf diesen Verkehr ergeben. Der im amerikanischen Sprachbereich übliche Gebrauch des Plurals „communications “ bezieht sich im allgemeinen auf die Revolutionierung der menschlichen Kommunikation durch die Benutzung von technischen Mitteln wie Presse, Film, Hörfunk und Fernsehen. Dies ist ein völlig neues Forschungsfeld, unserem technologischen Zeitalter eigentümlich zugehörig, $\mathrm{zu}$ dem vom heiligen Augustinus keine wesentlichen Beiträge erwartet werden können.

Kardinal König spricht vom Phänomen der „Verstärkung“ als typischem Ergebnis der amerikanischen Schule der Kommunikationsforschung. Gegen Ende seines Vortrages stellte er die Frage: „Was kann uns die moderne Kommunikationsforschung für die Aufstellung möglicher Strategien lehren? Vielleicht beginnen wir mit dem Hinweis auf ein Gesetz, das durch die Erforschung der Massenmedien erarbeitet worden ist. Nach diesem Gesetz neigen die Empfänger von Massenkommunikationen sehr stark dazu, nur solche Mitteilungen im Gedächtnis zu behalten, die Einstellungen verkörpern, mit denen sie schon sympathisieren..." Die Medienwirkung „besteht häufig nur darin, daß bereits vorhandene Überzeugungen verstärkt werden ". 5 
Doch dieses sogenannte Gesetz ist nur in Hinsicht auf Propagandakampagnen verifiziert worden. Die Kirche soll Lehrer, nicht Propagandist sein, und daher sollte sie pädagogische und nicht propagandistische Mittel in der Auslegung der Lehren des Evangeliums für die Menschheit benutzen.

Zudem wurde das „Verstärker"-Phänomen nur bei intensiven Propagandakampagnen kurzer Dauer bestätigt. Zum ersten Male wurde es beobachtet bei einer Studie, an der Lazarsfeld teilnahm: über die Wirkungen der Propaganda-Kampagne zur Präsidentschaftswahl von 1940 auf eine Gruppe von 600 Befragten in Eerie County, Ohio. Eine neue Untersuchung von Stanislas de Lestapis S.J. vom Zentrum für demographische Studien in Paris (vgl. „Relations“, Montreal, Juli-August 1967) legt den Schluß nahe, daß ein über Jahrzehnte und Generationen hinweg durchgeführtes Propaganda-Unternehmen Attitüden erheblich verändern kann. Nach dem Studium der Geburtenkontrolle-Propaganda in Frankreich während mehr als eineinhalb Jahrhunderten kommt Lestapis zu dem Ergebnis, daß der negative Charakter dieser Propaganda eine "Anti-Geburts"-Mentalität hat entstehen lassen, welche zu Angst und Ablehnung der Zeugung gegenüber geführt hat. Das Endergebnis war ein Meinungsklima, das der Abtreibung gegenüber günstig eingestellt war.

Bisher konzentrierte sich das theologische Interesse der Kirchen auf die Frage der Verantwortlichkeit bei der Benutzung der technologischen Macht der modernen Medien. Wir erwähnten den Schritt des Nationalen Kirchenrates in den USA, einen führenden Exponenten der amerikanischen Kommunikationsforschung zu beauftragen, ein Buch über Verantwortung und Massenmedien zu schreiben. Der Weltkirchenrat in Genf zeigte ein ähnlich gelagertes Interesse, indem er 1966 ein internationales Symposium abhielt über das Thema: "Die Antwort der Christen auf die Kommunikationsrevolution." Das Thema des Dekrets des Zweiten Vatikanischen Konzils über die publizistischen Mittel war ebenfalls die zivilisierte und christliche Anwendung dieser enorm machthaltigen Kommunikationsinstrumente. Der verantwortliche Gebrauch der Medien ist für die Zukunft der menschlichen Gesellschaft von größerer Bedeutung als der verantwortliche Gebrauch der nuklearen Macht. Kardinal König spielte auf das Konzils-Dekret zur Kommunikation in seinen ersten zwei Sätzen an und wandte sich dann abrupt und ohne Kommentar von diesem Thema ab. Wir nehmen an, daß der Kardinal dieses Dokument des Interesses der Theologen durchaus für würdig erachtet. Es ist bedauerlich, daß er es unterließ, das in seinem Vortrag ausdrücklich $\mathrm{zu}$ sagen.

Die hier und da zu beobachtende Diskreditierung des Dekrets über die publizistischen Mittel geschah nämlich oft durch so unbegründete und absurde Vorwürfe, daß ihre Autoren nie versuchten, sie zu rechtfertigen. Unkritisch übernommen von PaperbackHerausgebern, welche schnell den größten Marktanteil für Konzilsdokumente und -kommentare gewinnen wollten, wurden diese Angriffe weit verbreitet. Den echten Wert des Dekrets zu begründen, obliegt seinen Autoren - den Bischöfen, indem sie mutig seine Vorzüge hervorheben und sich zum darin beschlossenen apostolischen Programm bekennen.

Anmerkungen:

1. New York 1957

2. Wilbur Schramm (Hrsg.): The Science of Human Communication. New York und London 1963.

3. Glencoe 51965 (1. Aufl. 1960) 
4. Stanford, Cal., 1961

5. Franz Kardinal König: Kirche und Kommunikation, in: "Communicatio Socialis", Münster/Rom, 1. Jg. 1968, S. 6-20, hier S. 17.

\section{S U M M A R Y}

In his Toronto lecture on "Theology of Communications" (see CS 1:1, p. 6-20) Cardinal Koenig of Vienna urged theologians to interest themselves in a type of communications research, developed in the United States during the past 30 years, which, he thought, could aid the strategy of the Church in communicating with modern man. But the problem of American communications research (Lazarsfeld, Lasswell, Lewin, Hovland e. a.) is how to re-orient it to become an effort for the genuine benefit of society as a whole, rather than continuing to be a search for ever more ingenious methods of manipulating men's mind on behalf of the consumer goods industries. There are certainly many educators who would have appreciated it if Cardinal Koenig had urged universities to commit themselves seriously to the task of building up a better kind of communications research than that which seeks to develop more efficient techniques of propaganda. The Church is supposed to be a teacher, not a propagandist, and therefore her strategy should be to use pedagogic, not propagandistic, means in expounding the lessons of the Gospel to mankind. - The Cardinal alluded to the conciliar decree on communications only in his first two sentences. But the true worth of this decree has to be established by its authors, the bishops, committing themselves to the apostolic program it contains.

\section{R ES UMEN}

En la conferencia sobre el tema "Teología de las Comunicaciones ${ }^{\star}$ (Cf. CS 1:1, p. 6-20), que el Cardinal König, de Viena, tuvo en Toronto, urgió a los teólogos a que se interesaran en un tipo de investigación de comunicaciones, que se ha desarrollado en los Estados Unidos en los últimos 30 años, el cual, pensaba podría ayudar a la estratégia de la Iglesia en sus esfuerzos de ponerse en contacto con el mundo. Pero, el problema de investigación de comunicaciones americano (Lazarsfeld, Lasswell, Lewin, Hovland, etc.) trata de cómo reorientarlo para que se convierta en un genuino auxilio para el bienestar de toda la sociedad, en lugar de continuar siendo un centro de investigación para hallar métodos más ingeniosos de manipular la mente de los hombres, en beneficio de los industriales de bienes de consumo. Existen muchos educadores que hubieran preferido que, el Cardenal, hubiera hablado y exhortado a los estudiantes y a las universidades a dedicarse seriamente al establecimiento de un centro de investigación sobre las comunicaciones, en lugar de llamar la atención sobre un centro que sólo trata de desarrollar técnicas más refinadas de propaganda. La Iglesia es una Maestra, no una propagandista, $y$, por lo tanto, su estratégia debe consistir en poner en práctica la pedagogía, en lugar de la propaganda, cuando expone las lecciones del Evangelio a la humanidad. - El Cardenal aludió al Decreto Conciliar sobre las Comunicaciones sólo en sus dos primeras frases. Mas, el verdadero valor de este Decreto debe ser establido por sus autores, los Obispos, practicando el programa apostólico que contiene. 